Reacreations in Science and Mathematics

Ozanam

(Translated by Hutton; out of print; only second-hand copies obtainable)

Scientific Romances

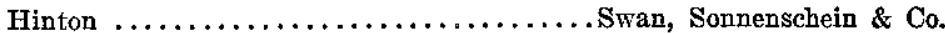

Fourth Dimension

Hinton $\ldots \ldots \ldots \ldots \ldots \ldots \ldots \ldots \ldots \ldots$. Swan, Sonnenschein \& Co.

F'latland

Anon..$\ldots \ldots \ldots \ldots \ldots \ldots \ldots \ldots \ldots \ldots$. Little, Brown \& Co.

Geometric Exercises in Paper Folding

Row $\ldots \ldots \ldots \ldots \ldots \ldots \ldots \ldots \ldots \ldots \ldots \ldots$ Open Court

How to Draw a Straight Line

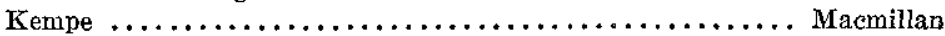

\title{
PROVISIONAL REPORT OF THE NATIONAL COMMITTEE OF FIFTEEN ON GEOMETRY SYLLABUS. ${ }^{2}$
}

\section{Problems Involving Loci.}

(a) Phraseology. While the committee does not wish to prescribe the exact phraseology of any definition, it would recommend greater care in the formulation of the definitions underlying the subject of loci. It is suggested that any definition used should be substantially equivalent to the following:

The locus of a point (or the locus of points) satisfying given conditions is a configuration such that:

(1) All points lying on the configuration satisfy the conditions;

(2) All points satisfying the conditions lie on the configuration.

It would seem desirable to make all proofs on loci conform to this definition. It is of course understood that the teacher will lead the pupil up to such a definition through varied forms of concrete description, such as "path of a point in motion," etc.

(b) Motion in geometry. It seems well to give some consideration to the place of motion in a well-rounded course in elementary geometry, and to bear in mind that this course is all the geometry to be studied by the majority of high school pupils. It has recently been urged by prominent European mathematicians that motion should be given a more prominent place at this stage. We may well recall that the space concepts dealt with in our usual courses in geometry are almost entirely to be described as static. There is in theorems and problems on loci a dynamic element that is of importance. The pupil is pretty fa-

INOTE. The Geometry report is completed in this issue. It has already been reprinted in pamphlet form and sent to two hundred selected teachers for the purpose of securing further suggestions and criticiems prior to its final presentation at the meeting of the National Educational Association at San Francisco in July. The Committee would invite suggestions and criticisms from any reader of SCHOOL SCIENCE AND MATHE. MATICS who may be interested. Such communications may be sent to any member of the Committee or directly to the Chairman, H. E. Slaught, 5535 Monroe Ave., Chicago, 111. 
miliar with motion as a concrete experience, and it seems of first-class importance to idealize some such concrete experiences, until they possess the precision of geometry.

For example, in a given plane we may consider in a way well described as a static configuration the perpendicular bisector of a line-segment joining two points; but when we consider this line as generated by a point moving in the plane in such a way that it is always equidistant from the two given points, we add a dynamic element.

As to phraseology, the expression "locus of points" suggests a static configuration, while the expression "locus of a point" emphasizes the dynamic element, and is equivalent in thought to the "path of a point moving with certain prescribed conditions." Both of these phases should have a place in the treatment of loci problems, and thus both forms of expression should be used, the one or the other being more suggestive in different cases. It is even desirable to use different forms in describing a given case to make clear the idea and to cultivate facility in expression.

(c) Concrete nature of loci. Contrary to the usual conception, the locus idea is one that may very easily be made concrete and brought down to the comprehension of young pupils. For example, the opening of a book or of a door suggests a variety of loci. The same may be said of many concrete illustrations easily accessible to the pupil.

In this way, loci problems may and should be introduced at certain stages of the strbject. For example, in Book I: The locus of a point equidistant from two fixed points, equidistant from two intersecting lines, or from two parallel lines, or at a given distance from a fixed line. In the book on circles, the locus of all points equidistant from a fixed point, the locus of the centers of circles of fixed radius and tangent to a given line, the locus of the centers of all circles tangent to two parallel lines or two intersecting lines, and the locts of the vertices of all triangles having a common base and equal vertex angles. In solid geometry, the locus of points equidistant from a given point, from two given points, from a given plane, from two intersecting planes, from two parallel planes, etc.

(d) Loci in problems of construction. Important features of the construction problems in geometry are dependent upon loci considerations which should be emphasized in this connection. For example:

(I) To find the locus in the plane of all points equidistant 
from three given points, it is necessary to determine the intersection of two loci both of which are straight lines.

(2) To find the locus in the plane of all points equidistant from a fixed point and at a given distance from a given line, it is necessary to find the intersection of two loci one of which is a straight line and the other a circle.

The discussion of the various possibilities in connection with stuch problems is one of the most valuable exercises for the pupil. For example, as to whether there are one, two, or no points fulfilling the conditions in the second example above. While it may be possible to solve and discuss such problems without using the term locus at all, yet this leads to roundabout and awkward explanations while the language of loci is elegant and concise.

Moreover, facility in the use of this language is not only desirable from the standpoint of the high school pupil but is of the utmost importance for those who may continue the study of geometry in college.

(e) To summarize, the locus idea is deserving of a careful and systematic treatment for the following reasons:

(1) It introduces a dynamic element through the consideration of the idea of motion.

(2) It presents an elegant language for the statement of those propositions on which nearly all of our problems of construction are based.

(3) It aids greatly in the cultivation of space intuition and in emphasizing the important concept of functionality.

\section{(f) Additional illustrations appropriate for use:}

1. Find the locus of all points at a fixed distance from the sides of a triangle, always measuring from the nearest point of a side.

2. Find the locus of points such that the sum of the squares of the distances from two lines intersecting at right angles is 100 .

3. Find the locus of the vertices of a regular polygon of a given number of sides that can be circumscribed about a given circle.

4. Find the locus of the midpoints of the sides of regular polygons of a given number of sides that can be inscribed in a given circle.

5 . Find the locus of all points from which a given line-segment subtends a given angle.

6. Find the locus of a point the sum of the squares of whose distances from two given points is constant.

7. Find the locus of a point the difference of the squares of whose distances from two given points is constant.

8. Find the locus of all lines drawn through a given point; parallel to a given plane.

9. Find the locus of a point in space equidistant from three given points not in a straight line. 


\section{Algebraic Methods in Geometry.}

The committee feels that the use of algebraic forms of expression and solution in the geometry courscs may well be extended, with advantage to both algebra and geometry, and that this may be done without in any way encroaching upon the field of analytic geometry, which belongs to a later stage of development.

(a) The notation should be more algebraic. While it is not feasible or desirable to lay down hard and fast rules to standardize the notation of geometry, an examination of current texts makes it evident that some notations in common use are unnecessarily awkward when compared with the notations used in elementary algebra. The notation of geometry is, in general, improved by much use of lower-case letters to represent numerical values, leaving capitals to represent points. This notation is here called algebraic because the student will recognize the relations of equality and inequality much more readily in the familiar notation of algebra than if these relations are presented in a notation not used in algebra.

(b) Algebraic statement of propositions. Many of the theorems cf geometry may be stated to advantage in algebraic form, thus giving definiteness and perspicuity and especially emphasizing the notion of functionality. This mode of expression can be made of much value to the student if he is required to translate into English all the symbols involved.

The following are illustrations of the algebraic statement of propositions:

(1) In any triangle, $a=b h / 2$, where $a$ is the area, $b$ is the base and $h$ is the altitude.

(2) In a right triangle, $c^{2}=a^{2}+b^{2}$, where $c$ is the hypotenuse; and $a$ and $b$ are the sides including the right angle.

(3) In any triangle $c^{2}=a^{2}+b^{2} \pm 2 a p$, where $a, b, c$ are sides of the triangle and $p$ is the projection of $b$ on $a$.

(4) For any secant and tangent drawn from a point to a circle, we have $t^{2}=s x$, where $t$ is the length of the tangent, $s$ is the length of the secant and $x$ is the length of the external part.

It is not intended to convey the impression that the usual statement of propositions should be replaced by the algebraic statements but rather that the student should be required to translate the one form of statement into the other. The algebraic statements are often superior to the usual statements in point of brevity and conciseness. Moreover, the algebraic statement prepares for the idea of functionality which is too little understood by persons who are not trained in mathematics be- 
yond the high school course. That is to say, some appreciation of the influence of changing one part of a configuration on other parts of the configuration can often be gained readily from the algebraic statement.

(c) Geometrical construction of formulas. Some propositions can be proved simply and elegantly by methods involving algebra. It is somewhat usual in text-books on geometry to give a proof of the geometrical statement of such an algebraic formula as $(a+b)^{2}=a^{2}+b^{2}+2 a b$, where $a$ and $b$ are the numerical measures of the line segments, but to neglect the geometrical construction of the formula. The latter seems to be the point of greatest importance. It is not additional evidence of the validity of the theorem that is sought. That is established in algebra. What is of first-rate importance is to give a geometrical picture of the formula, thus showing a certain geometrical interpretation and to have the student put the result into geometrical phraseology when $a$ and $b$ are line-segments.

The construction of line-segments $a+b, a-b$, and of areas $a b,(a+b)^{2},(a-b)^{2}$, where $a$ and $b$ are line-segments should come early in the course. Later, when the requisite theorems are being developed, the further elementary expressions

$$
k a, \frac{a}{k}, \frac{a b}{c}, \sqrt{a b}, \sqrt{a^{2}+b^{2}}, \sqrt{a^{2}-b^{2}}, a \sqrt{k},
$$

where $a, b$, and $c$ are line-segments and $k$ is a positive integer, should be constructed.

This interdependence of algebra and geometry is a matter of no small importance both historically and for subsequent mathematical work. It should be brought out by suitable exercises that the use of algebra often enables one to establish relations from which a geometrical construction can be made readily or to show the nature of a difficulty involved.

For example, to inscribe a square in a semicircle:

If $x$ represents the side of the square and $r$ the radius of the circle, we have at once from a right triangle that $r^{2}=x^{2}+x^{2} / 4$ and hence $x= \pm 2 / 5 \sqrt{5} r$, which can be constructed from exercises given above.

(d) Geometric exercises for algebraic solution. Some exercises for algebraic solution, such as are found in many recent texts, should find a place in any course in geometry. For example, the following is a suitable exercise after the proposition stating that $a=b h$, where $A$ is the area, $b$ and $h$ are sides of a rectangle: 
The area of a rectangle is 480 square inches. Each side of the rectangle is increased 1 inch and, by this change, the area is increased 45 square inches. Find the sides of the rectangle.

Similarly, after the proposition pertaining to secants and tangents to a circle, the following is stitable:

A secant line which passes through the center of a circle of radius 10 is intersected by a tangent of length 15. Find the length of the external part of the secant.

Such exercises do much to unify geometry and algebra, and may well replace some of the usual exercises.

Finally, after the theorem on the volume of a frustum of a pyramid, a problem like the following has value as an algebraic exercise, although it is in no sense a real applied problem.

A pier is built of solid concrete construction, in the form of a frustum of a pyramid with square bases. The altitude is twice an edge of the lower base and the area of the Iower base is four times that of the upper base. Find the dimensions of each base if the pier contains 600 cubic feet of solid concrete.

\section{SEOTION E. SYLLABUS OF GEOMETRY.}

PREFACE TO LISTS OF THEOREMS.

(I) Lists not exhaustive. The lists of theorems which follow are not to be taken as exhaustive, and it is distinctly understood that theorems may be added at the discretion of the teacher. For example, the theorem on the existence of regular polyhedra may find a place in certain courses. Some theorems are omitted only with the understanding that they may be inserted as exercises for the student; some such possible exercises are:

In any triangle, the product of any two sides is equal to the product of the segments of the third side formed by the bisector of the opposite angle, plus the square of the bisector.

Lpon a given line-segment corresponding to a given side of a given polygon, to construct a polygon similar to the given polygon.

To divide a given straight line-segment in extreme and mean ratio.

To find the area of a triangle in terms of its sides.

To construct a square having a given ratio to a given square.

The surface of a sphere is equivalent to the area of four great circles.

(2) Logical order. Although there is some indication of a possible logical order in the lists, there is no intention of specifying any definite order. It would be impossible to carry out as a whole precisely the order stated below.

It should be noticed that some logical arrangements would necessitate the insertion of the theorems omitted in this list. 
Such an insertion is entirely in the spirit of this report, as is also any conceivable change in the order, except where specified explicitly in the report.

(3) Subsidiary theorems. A number of theorems omitted in the lists below may well be given as ordinary statements in the course of the text as corollaries, or as remarks, without the emphasis which attaches to formal theorems. Among such general statements which should by all means be made at the proper points are the following:

No triangle can have more than one right angle or more than one obtuse angle.

The third angle of a triangle can be found if two are known.

An equilateral triangle is equiangular.

The square on a side of a right triangle adjacent to the right angle is equal to the square on the hypotenuse minus the square on the other side.

Through three points not in a straight line not more than one plane can be passed.

The areas of two spheres are to each other as the squares of their radii; their volumes as the cubes of their radii; (like statements for other solids).

The number of such statements is exceedingly large and all of them could not be given in any syllabus. A large majority are at the present time stated, if at all, in the coturse of the reading matter, or in exercises, and not as explicit theorems. It is understood, and indeed expected, that these statements, together with many which are omitted from the lists of theorems below, should be treated in this manner.

(4) Informal proofs. The theorems given below under the heading: "Theorems for informal proofs," should be stated at the proper points in the text and in theoren form, or as postulates. Their proofs, however, can well be omitted where this omission is suggested, or be made exceedingly informal by the insertion of a single phrase which will give the proper suggestion for the proof. Many other theorems which are equally obvious are not stated because they occur more naturally as corollaries or as exercises. (See the preceding paragraph.)

Regarding the method of proof in general, while the demonstrations should remain as logical as they are at present, it is suggested that the formalities of logic, as such, be frequently dispensed with to a very considerable extent and that the propositions be frequently stated and proved in language resembling that to be found in any other mathematical text-book. This is, indeed, the style of many classical treatises, such as Legendre's or. Euclid's. It is certainly satisfactory and there is no reason 
why the proof should not remain quite as logical when the older style is followed.

The symbolic form of demonstration which appears in many texts should be regarded simply as a shorthand expression of a complete proof in ordinary English phraseology. The latter should be given by the student in all cases. The ability to pass from the symbolic form to ordinary English, that is, to translate the shorthand into the language of everyday life, should be constantly tested by the teacher, for the same reason that the formulas of algebra derive their real meaning and power from the thought content which the student can attach to them.

(5) Arrangement for emphasis. The main list of theorems is divided into several heads, each group being introduced by a theorem of suitable importance upon which the rest of the theorems in that group depend more or less closely. This arrangement has been selected in order to emphasize the importance of a few major propositions, namely, those which carry a maximum of applications and from which the rest can be derived, thus serving as a nucleus for the whole of geometry.

This effort to gain emphasis has been carried out still further by printing the theorems in different grades of type so that those of fundamental importance and of basal character are printed in black face type; those of considerable importance which are secondary only to the preceding ones are printed in italics. A number of other theorems are printed in Roman type, while the least important are printed in small type. The latter (small type theorems) may be omitted without serious danger, or they may be used as corollaries or exercises instead of receiving the emphasis which attaches to a theorem; in fact, probably no injury would result from a similar treatment of many of the theorems stated in Roman type.

The distinction in emphasis is desirable not only for guidance in omitting theorems in courses which are necessarily abbreviated, but it is also of the highest importance in courses in which all of the theorems are given. An orderly classification of theorems in the student's mind, a notion of the dependence of the minor theorems on the more basal ones and an appreciation of their relative importance is of the utmost direct value to the student and furnishes him with the only possible means of permanently retaining geometrical knowledge in usable form. The direct value mentioned arises both from the power acquired and also from the essential grasp of the subject, which 
is the purpose of education. It is the fundamental characteristic of the human mind from which there is no escape that any impression of a vast field must have exactly such distinctions in emphasis as are outlined here for geometry. These statements and this arrangement are intended to be of assistance to the teacher in guiding him as to the emphasis to be laid upon theorems during the course and especially at the completion of a given book or chapter.

(6) Trigonometric ratios. Attention is called to the paragraphs under XIII, 2-4 on the computation of two-place tables of sines, cosines, and tangents from actual measurements, provided the pressure of time due to examining bodies is not too great. This work can be done with about the same amount of effort that is expended by the student on the ordinary geometrical theorems of the same class. Its importance is due to the fact that such a small table will really present the fundamental ideas of trigonometry and will enable the student to solve right triangles in the trigonometric sense.

(7) Abbreviations. In a large number of instances theorems are stated in condensed or abbreviated form and the statement of a number of theorems is often combined into one. This is done only for the purpose of reducing the length of this report. It is to be understood that such abbreviated statements are made only for the teacher and should not be presented to the student in this form. In particular, it is probably preferable to use words instead of letters in statements for high school pupils of such theorems as those in II, I-4.

The numbers which follow each of the theorems are references to a syllabus prepared by a committee of the Association of Mathematics Teachers of New England, 1906.

(8) Omissions. The following list shows the omissions from the New England Syllabus:

Plane geometry omissions: $\mathrm{C} 2, \mathrm{G} 12$ (2nd part), G20, Jı3, L2, N6(2nd part), Ni3, Pr, P2 (see note to XV, I), P6, PI2, T2, $\mathrm{T}_{4}, \mathrm{~T}_{5}$.

Solid geometry omissions: E7, E8, E9b, FI2, Fi3, Fi4, $\mathrm{K}_{2}$, $\mathrm{K}_{3}$ (see note to $\mathrm{I}, 2$ ), M6, M7, M8, M9, Q3, Q4, Q8b, Q9 (see VI, note), Rx, R2 (see note to VI, I3), R9, Rio, Ri3, Ri4 (see note to $\mathrm{VI}, 22$ ), $\mathrm{S}_{3}$ (see preface, 3 ), $\mathrm{S}_{7}$. 
THEOREMS OF PLANE GEOMETRY.

\section{Theorems for Informal Proof.}

(The following theorems may be stated as assumptions, or may be given such informal proof as the circumstances may demand.)

1. All straight angles are equal. ${ }^{45}$ [\%]

2. All right angles are equal. [*]

3. The sum of two adjacent angles whose exterior sides lie in the same straight line equals a strajght angle. [J1.]

4. If the sum of two angles equals a straight angle their exterior sides form a straight line. [J2.]

5. Only one perpendicular can be erected from a given point in a given line. [G3.]

6. The length of a circle (circumference) lies between the lengths of perimeters of the inscribed and circumscribed convex polygons. [P13.]

(It is recommended that this statement be used as a definition to be inserted at context.)

7. The area of a circle lies between the areas of circumscribed and inscribed convex polygons. [P14.]

(It is recommended that this statement be used as a definition to be inserted at context.)

8. Two lines parallel to the same line are parallel to each other. $[*]$

9. Vertical angles are equal. [JJ3.]

(Very informal proof sufficient.)

Io. Complements of equal angles are equal. [N2.]

I I. Supplements of equal angles are equal. [N3.]

I2. The bisectors of vertical angles lie in a straight line. [J4.]

I3. Any side of a triangle is less than the sum of the other two and greater than their difference. [*]

14. A diameter bisects a circle. [A5.]

15. A straight line intersects a circle at most in two points. [G6.]

\section{Congruence of Triangles.}

\section{Any two triangles ${ }^{46} \mathbf{A B C}$ and $\mathbf{A}^{\prime} \mathbf{B}^{\prime} \mathbf{C}^{\prime}$ are congruent if:}

\begin{tabular}{|c|c|c|c|c|}
\hline (1) & $\mathrm{a}=\mathrm{a}^{\prime}$ & $\mathrm{b}=\mathrm{b}^{\prime}$ & $\mathbf{c}=\mathbf{c}^{\prime}$ & [A1.] \\
\hline (2) & $a=a^{\prime}$ & $\mathbf{B}=\mathbf{B}^{\prime}$ & $\mathbf{C}=\mathbf{C}^{\prime}$ & [A2.] \\
\hline (3) & $a=a^{\prime}$ & $\mathrm{b}=\mathrm{b}$ & $\mathrm{c}=\mathrm{c}^{\prime}$ & [A3.] \\
\hline (4) & $a=a^{\prime}$ & $\mathbf{c}=\mathbf{c}^{\prime}$ & $\mathbf{c}=\mathbf{c}^{\prime}$ & [A4.] \\
\hline
\end{tabular}

(State these in detail and in English. See preface, article 7.)

2. A triangle is determined when the following are given: (I) a, b, C; (2) a, B, C; (3) a, b, c; (4) a, c, C $=90^{\circ} . \quad[*]$ (Synonymous to 1 .)

${ }^{45}$ Reference numbers are to the New England Syllabus. Where an asterisk [*] replaces the reference number, the theorem is not contained in that syllabus.

${ }^{4}$ In this syliabus the angles of a triangle ABC ate denoted by the capital letters $A, B$, and $C$; the sides are denoted by small letters $a, b$, and $c$, where $a$ is the side opposite the angle $A$, etc. 
3. Construction of triangles from given parts; measurement of unknown parts by ruler and protractor. Given: (I) $a, b, \mathrm{C}$; (2) $a, \mathrm{~B}, \mathrm{C}$; (3) $a, b, c$; (4) $a, c, \mathrm{C}$, possibly two solutions. [*] (This is the fundamental, elementary idea of trigonometry.)

4. In any two triangles, if $a=a^{\prime}$ and $b=b^{\prime}$, either of the inequalities $c>c^{\prime}$ or $\mathrm{C}>\mathrm{C}^{\prime}$ is a consequence of the other. [03, 04.]

\section{Congruent Right Triangles.}

1. Two right triangles are congruent if, aside from the right angles, any two parts, not both angles, in the one are equal to corresponding parts of the other. [A4.]

(Very important: subcase of II, 1.)

2. If two oblique lines $c$ and $c^{\prime}$ be drawn from a point in a

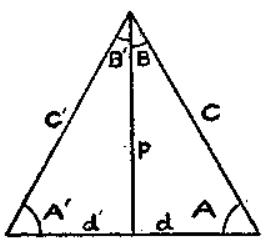
perpendicular $p$ to a line $\mathrm{AA}^{\prime}$, cutting off distances $d$ and $d^{\prime}$, then any one of the equalities, $c=c^{\prime}, d=d^{\prime}, \mathrm{A}=\mathrm{A}^{\prime}, \mathrm{B}=\mathrm{B}^{\prime}$, is a consequence of any other. [G5.]

3. A diameter perpendicular to a chord bisects the chord, the subtended angle at the center, and the subtended arc; conversely, a diameter which bisects a chord is perpendicular to it. [G5b, G8.]

(Corollary to 2. See also IV, 3.)

4. If two oblique lines $c$ and $o^{\prime}$ be drawn from a point in a perpendicular $p$ to a line $\mathrm{AA}^{\prime}$, cutting off unequal distances $d$ and $d^{\prime}$, then either of the inequalities $c>c^{\prime}, d>d^{\prime}$, is a consequence of the other. [05, 06.]

(In particular, $e$ is greater than $p$.)

5. If, in a triangle $A B C, a=b$, the perpendicular from $C$ on $c$ divides the triangle into two congruent triangles. [*]

6. In a triangle $A B C$, either of the equations $a=b, A=B$, is a consequence of the other. [G1, G2.]

7. In a triangle ABC, either of the statements $a>b, A>B$, is a consequence of the other. [01, 02.]

\section{Subtended Arcs, Angles and Chords.}

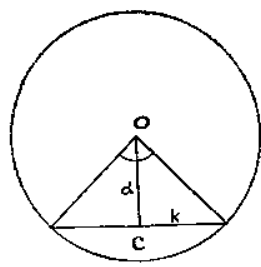

1. In the same circle, or in equal circles, any one of the equations $d=d^{\prime}, k=k^{\prime}, c=c^{\prime}$, $0=0^{\prime}$, is a consequence of any other one of them. $[\mathrm{A} 6,7,8,9, \mathrm{G} 9$.

2. Any one of the inequalities (see figure), $d<d^{\prime}, \quad 0>0^{\prime}, c>c^{\prime}, k>k^{\prime}$,

is a consequence of any other one of them. $[07,8$.

3. In any circle an angle at the center is measured by its intercepted arc.

(Only the commensurable case.)

4. If a circle is divided into equal arcs, the chords of these arcs form a regular polygon. [G12.] 
5. To construct an angle equal to a given angle. [J14.]

(Regular polygons may be constructed approximately by means of a protractor. In the same way other approximate constructions may be introduced which depend upon the protractor.)

\section{Perpendicular Bisectors.}

1. The perpendicular bisector of a line-segment is the locus of points equidistant from the ends of the segment. [S1.]

2. To draw the perpendicular bisector of a given line-segment. [G14.]

3. To erect a perpendicular at a given point in a line [*]

(Corollary to 2.)

4. To drop a perpendicular from a given point to a given line. [D5.]

(Corollary to 2.)

5. To bisect a given arc or angle. [G15, 16.]

(See III, 3.)

6. To inscribe a square in a circle. [G18.]

7. One and only one circle can be circumscribed about any triangle. [G7.]

(Construction to be given.)

8. Three points determine a circle. Two circles can intersect, at most, in two points; this will happen when the distance between their centers is less than the sum of the radii and greater than the difference of the radii. [G7.]

\section{(Corollary to 7. )}

9. Given an arc of a circle, to find its center. [*]

(Corollary to 7.)

10. A circle may be circumscribed about any regular polsgon. [G13, third part.]

11. The perpendicular bisectors of the sides of a triangle meet in a point. [T3.]

(Corollary to 7.)

\section{Bisectors of Angles.}

1. The bisector of any angle is the locus of points equidistant from the sides of the angle. [S2.]

2. A circle may be inscribed in any triangle. [G13, second part.]

(Construction to be given.)

3. A circle may be inscribed in any regular polygon. [G13, last part.]

4. Of the inscribed and circumscribed regular polygons of $n$ and $2 n$ sides for a given circle, to draw the remaining three polygons when one is given. [G17.]

5. The bisectors of the angles of any triangle meet in a point.

(Corollary to 2.)

VII. Parallels.

1. When two lines are cut by a transversal the alternate interior angles are equal if, and only if, those two lines are parallel. [Half of D1, 2.] 
When two lines are cut by a transversal, the alternate interior angles are unequal, if and only if, the lines are not parallel.

(Synonymous to 1. .)

2. When two lines are cut by a transversal the corresponding angles are equal, and the two interior angles on the same side of the transversal are supplementary if, and only if, the two lines are parallel. [Half of D1, 2.]

(Corollary to 1.)

3. Two lines perpendicular to the same, line are parallel. [D4, G4.]

(Only one perpendicular can be let fall from a point without a line to that line. Synonymous to 3.)

4. A line perpendicular to one of two parallels is perpendicular to the other also. [D3.]

(Corollary to 1.)

5. If two angles have their sides respectively parallel or respectively perpendicular to each other, they are either equal or supplementary. [J].]

6. Through a given point to draw a straight line parallel to a given straight line. [D6.]

7. A parallelogram is divided into two congruent triangles by either diagonal. [*]

8. In any parallelogram the opposite sides are equal, the opposite angles are equal, the diagonals bisect each other. [D7.]

(Corollary to $\boldsymbol{T}$.)

9. In any convex quadrilateral, if the opposite sides are equal, or if the opposite angles are equal, or if one pair of opposite sides are equal and parallel, or if the diagonals bisect each other, the figure is a parallelogram. [D8.]

\section{Angles of a Triangle.}

1. In any triangle the sum of the angles is two right angles. [J5(b).]

2. In any triangle, any exterior angle is equal to the sum of the two opposite interior angles. [J5(a).]

(Corollary to 1.)

3. The sum of the interior angles of any polygon of $n$ sides is $2(n-2)$ right angles. [J6.]

4. To inscribe a regular hexagon in a circle. [G19.]

To construct an angle of $60^{\circ}$. (Synonymous to 4 .)

\section{Inscribed Angles.}

1. An angle inscribed in a circle is measured by half of its intercepted arc. [J9.]

2. Angles inscribed in the same segment are equal to each other. $[\%]$

3. An angle inscribed in a semicircle is a right angle. [*] 
4. The two arcs intercepted by parallel secants are equal. [G11.]

5. The angle between a tangent and a chord is measured by half the intercepted arc. [J10.]

6. The angle between any two lines is measured by half the sum, or half the difference, of the two arcs which they intercept on any circle, according as their point of intersection lies inside of, or outside of, the circle. $[J 11,12$.

7. The tangent to a circle at a given point is perpendicular to the radius at that point. [L1, 3.]

8. For a given chord, to construct a segment of a circle in which a given angle can be inseribed. [J15.]

9. To draw a tangent to a given circle through a given point. [L4.]

Io. The tangents to a circle from an external point are equal. [G10.]

(Corollary to 7 ).

\section{Segments Made by Parallels.}

1. If a series of paraliel lines cut off equal segments on one transversal, they cut off equal segments on any other transversal. [D9.]

2. The segments cut off on two transversals by a series of parallels are proportional. [See N10.]

(Only the commensurable case.)

3. A line divides two sides of a triangle proportionally, the segments of the tro sides being taken in the same order, if and only if it is parallel to the third side. [N1, 2.]

(Only the commensurable case.)

4. To divide a line-segment into $n$ equal parts or into parts proportional to any given segments. $[\mathrm{N} 9,10$.

5. To find a fourth proportional to three given line-segments. [N11.]

6. To find a mean proportional between two given line-segments. [N12.]

XI. Similar Triangles.

1. Two triangles $A B C$ and $A^{\prime} B^{\prime} C^{\prime}$ are similar if

(1) $\quad \mathbf{A}=\mathbf{A}^{\prime} \quad \mathrm{B}=\mathrm{B}^{\prime} \quad \mathrm{C}=\mathbf{C}^{\prime}$

or (2) $\mathrm{a}=\mathrm{ka}^{\prime} \quad \mathrm{b}=\mathrm{kb}^{\prime} \quad \mathbf{C}=\mathbf{C}^{\prime} \quad$ [N4.]

or (3) $\quad \mathrm{a}=\mathrm{ka}^{\prime} \quad \mathrm{b}=\mathrm{kb}^{\prime} \quad \mathbf{c}=\mathbf{k c}^{\prime} \quad$ [N5.]

where $\mathrm{k}$ is a constant factor of proportionality.

(See preface, article 7.)

2. Given a fixed point $P$ and a circle $C$, the product of the two distances measured along any straight line through $P$, from 
$P$ to the points of intersection with $C$, is constant. This product is also equal to the square of the tangent from $P$ to $C$ if $P$ is an external joint. [N18.]

3. The bisector of any angle of a triangle divides the opposite side into segments proportional to the adjacent sides. [Half of N6.]

4. To construct a triangle similar to a given triangle. [*]

(Drawing triangles to scale; measurements of remaining parts to scale. Basal in trigonometry.)

\section{Similar Figures.}

I. Polygons are similar if and only if they can be decomposed into triangles which are similar and similarly placed. [N7, 8.]

2. Regular polygons of the same number of sides are similar. [N14.]

3. The perimeters of similar polygons are proportional to any two corresponding lines of the polygons. [N15.]

4. The circunferences of any two circles are proportional to their diameters, thus, $c=2 \pi r$, where $\pi$ is constant. [P15.] ( $x=3.14 \ldots$ to be computed later.)

5. To construct a polygon similar to a given polygon. [*]

(Drawings to scale; xaps, house plans; readings from drawings; plotting of measurements. Essential in surveying.)

\section{Similar Right Triangles.}

(The committee feels that numbers $2,3,4$ following should have a place where time for their discussion can be secured, which will doubtless be the case except unter pressure from examining bodies.)

1. Any two right triangles are similar if an acute angle of the

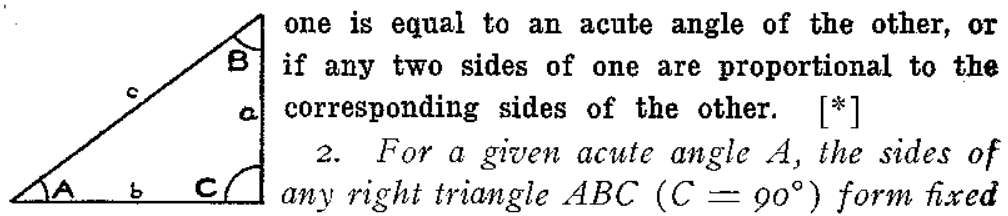
ratios, called the sine $(a / c)$, the cosine $(b / c)$, the tangent $(a / b)$. [*]

3. Computation of a two-place table of sines, cosines, tangents from actual measurements. [*]

(Probably a two-place table for every $5^{\circ}$ or $10^{\circ}$; to be done by students, preferably on squared paper.)

4. Solution of right triangles with given parts by use of the preceding table of ratios. [*]

(Height and distance exercises.) 
XIV. Right Triangles.

1. In any right triangle $A B C$ the perpendicular let fall from the right angle upon the hypotenuse divides the triangle into two similar right triangles, each similar to the original triangle. [\%]

2. The length of the perpendicular $p$ is the

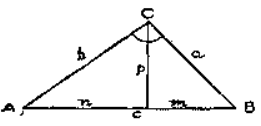
mean proportional between the segments $m$ and $n$ of the hypotenuse; i. e., $p^{2}=m n$. [P8.]

3. Either side, $a$ or $b$, is the mean proportional between the whole hypotenuse $c$ and the adjacent segment $m$ or $n$; that is, $a^{2}=c m ; b^{2}=c n$. [P9.]

4. The sum of the squares of the two sides of a right triangle is equal to the square of the hypotenuse; $\mathrm{a}^{2}+\mathrm{b}^{2}=\mathrm{c}^{2}$. [P10.]

(It should be noticed that the proposition can be proved either algebraically or geometrically.)

5. In any triangle $\mathrm{ABC}$, if $\mathrm{B}$ is less than $90^{\circ}$, then $b^{2}=a^{2}+c^{2}-2 c m$; if $\mathrm{B}$ is greater than $90^{\circ}$, then $b^{2}=a^{2}+c^{2}+2 c m$, where $m$ is the projection of $a$ on $c . \quad[\mathrm{P} 17$.

(See figure under 2.)

6. Given the radius of a circle and a perimeter of an inscribed regular pulygon of $n$ sides, to find the perimeter of the regular circumscribed polygon of $n$ sides and the perimeter of the regular inscribed polygon of $2 n$ sides. [*]

7. To calculate $\pi$ approsimately. [*]

\section{Areas.}

1. The area of a rectangle is the product of its base and its altitude; i. e., $a=$ bh. $[\mathrm{P} 1,2,3$.

(This formula may be taken as the definition of area.)

2. The area of a parallelogram is the product of its base and its altitude; i. e., $a=b h . \quad[\mathbf{P} 4$.

3. The area of a triangle is one half the product of its base and its altitude; i.e., $a=1 / 2$ bh. [P5.]

4. Parallelograms or triangles of equal bases and altitudes are equivalent. [C1.]

5. The area of a trapezoid is one half the product of its altitude and the sum of its bases; $i . e ., a=1 / 2\left(b_{1}+b_{2}\right) h$. [P7.]

6. The areas of similar triangles or polygons are proportional to the squares of corresponding lines. [N16, 17.]

7. The area of a regular polygon is one half the product of its perimeter and its apothem. [P11.]

8. The area of any circle is one half the product of its circumference and its radius; i. e., $a=\pi r^{2}$. [P16.]

9. The areas of two circles are proportional to the squares of their radii. [*]

(May be treated as suggested in preface, article 3. ) 

[*]

10. To construct a square equivalent to the sum of two given squares.

(Pythagorean proposition.)

11. To construct a square equivalent to a given rectangle.

(Mean proportional. See $\mathrm{X}, 6$.)

[C3.]

\section{THEOREMS OF SOLID GEOMETRY.}

In this part the same general principles apply as were stated in the preface above.

Throughout, but particularly in divisions I and II below, very great emphasis should be laid upon the student's real grasp of the conceptions, of the space figures, and of the significance of the theorems. While the theorems in division I will be seen to need little or no suggestion of proof, it is a mistake to suppose that they can be hastened over; on the contrary, even in these, the teacher should spare no pains to make sure that the student's mental picture is quite vivid, resorting to formal proof when necessary. To this end, illustrations, figures, models, various forms of presentation, and all such aids are legitimate throughout the course in solid geometry.

\section{Theorems for Informal Proof.}

I. If two planes cut each other, their intersection is a straight line. [S4.]

2. Two dihedral angles have the same ratio as their' plane angles. $[\mathbf{K} 2,3,4$.

(Equivalent to K3.)

3. Every section of a cone made by a plane passing through its vertex is a triangle. [M4.]

4. Every section of a cylinder made by a plane passing through an element is a parallelogram. [M2.]

5 . The area of a sphere lies between the areas of circumscribed and inscribed convex polyhedrons. [*]

(It is recommended that this statement be used as a definition to be inserted at context.)

6. The volume of a sphere lies between the volumes of circumscribed and inscribed convex polyhedrons. [*]

(It is recommended that this statement be used as a definition to be inserted at context.)

7. The projection of a straight line upon a plane is a straight line.

\section{Corollaries from Plane Geometry.}

(The ability to make the transfer from plane geometry to solid geometry, and vice versa, in forming conceptions and in logical deductions is of the utmost importance. The following 
theorems are easily reducible to plane geometry in at most two or three planes. The intention is that careful proofs be given, but the student should see that these theorems result immediately from known theorems of plane geometry.)

I. The intersections of two parallel planes with any third plane are parallel. [F1.]

2. A plane containing one and only one of two parallel lines is parallel to the other. [F7.]

3. If a straight line is parallel to a plane, the intersection of the plane with any plane drawn through the line is parallel to the line. $[*]$

4. Through a given point only one plane can be passed parallel to two straight lines not in the same plane.

(Derived from 2.)

5. Through a given straight line only one plane can be passed parallel to any other given straight line in space, not parallel to the first. [F11.]

(Derived from 2.)

6. Through a given point only one plane can be drawn parallel to a given plane. [F9.]

(Synonymous to 4.)

7. If a perpendicular $\mathrm{PO}$ be let fall from a point $\mathrm{P}$ to a plane $L$, any one of the equalities

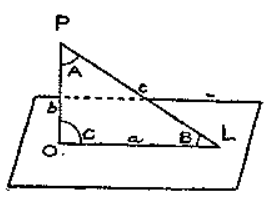

$$
a=a^{\prime}, c=c^{\prime}, \mathrm{B}=\mathrm{B}^{\prime}, \mathrm{A}=\mathrm{A}^{\prime}
$$

is a consequence of any other of them; and any one of the inequalities

$$
a>a^{\prime}, c>c^{\prime}, \mathrm{B}<\mathrm{B}^{\prime}, \mathrm{A}>\mathrm{A}^{\prime}
$$

is a consequence of any other one of them.

[010, H7. See also $\mathbf{5 8 .}]$

8. The perpendicular $\mathrm{PO}$ is shorter than any oblique line. [09.]

9. Two straight lines are parallel to each other if and only if they are both perpendicular to some one plane. $[\mathbf{F} 2,3$.

Io. If two straight lines are parallel to a third, they are parallel to each other. [F4.]

(Derived from 9.)

II. Two planes are parallel to each other if and only if they are both perpendicular to some one straight line. [F5, 6.]

(Derived from 9.)

r2. The locus of points equidistant from the extremities of a straight line is a plane perpendicular to that line at its middle point. [S5.] 
I3. If two straight lines are intersected by three parallel planes, their corresponding segments are proportional. [See M1.]

(Draw the traditional auxiliary figure.)

I4. The locus of points equidistant from two intersecting planes is the figure formed by the bisecting planes of their dihedral angles. [S6.]

\section{Planes and Lines.}

1. If a straight line is perpendicular to each of two other straight lines at their point of intersection, it is perpendicular to every line in their plane through the foot of the perpendicular. [E1.]

2. Every perpendicular that can be drawn to a straight line at a given point lies in a plane perpendicular to the line at the given point. [E2.]

(Corollary to 1.)

3. Through any point only one plane can be drawn perpendicular to a given line. [E5.]

(Corollary to 1 , and II, 11.)

4. Through a given point only one perpendicular can be drawn to a given plane. [E6.]

(Corollary to 1.)

5. If two angles have their sides respectively parallel and lying in the same direction, they are equal, and their planes are parallel. [K1, F8.]

6. If a line meets its projection on a plane, any line of the plane perpendicular to one of them at their intersection, is perfondicular to the other also. [*]

7. Between any two straight lines not in the same plane, one and only one common perpendicular can be drawn, and this common perpendicular is the shortest line that can be drawn between the two lines. [F12.]

8. Two planes are perpendicular to each other if and only if a line perpendicular to one of them at a point in their intersection

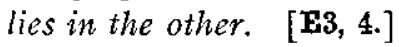

9. If a straight line is perpendicular to a plane, every plane passed through the line is perpendieular to the first plane. [E9a.]

(Corollary to 8 .)

10. If two intersecting planes are each perpendicular to a third plane, their intersection is also perpendicular to that plane. [E10.]

(Corollary to 8.)

II. Through a given straight line oblique to a plane, one and only one plane can be passed perpendicular to the given plane. [E11.]

12. The acute angle which a straight line makes with its own projection on a plane is the least angle which it makes with any line of the plane. [013.] 
13. Two right prisms are congruent if they have congruent bases and equal altitudes. [B1.]

I4. If parallel planes cut all the lateral edges of a pyramid, or a prism, the sections are similar polygons; in a prism, the sections are congruent; in a pyramid, their areas are proportional to the squares of their distances from the vertex. [M1.]

(See II, 13.)

15. Every section of a circular cone made by a plane parallel to its base is a circle, the center of which is the intersection of the plane with the axis. [M5.]

16. Parallel sections of a cylindrical surface are congruent. [M3.]
[M]

\section{Spheres.}

1. Every section of a sphere made by a plane is a circle. [M10.]
[Med (Several corollaries may be added.)

2. The intersection of two spheres is a circle whose axis is the line of centers. [H4.]

3. The shortest path on a sphere between any two points on it is the minor arc of the great circle which joins them. [014.]

4. A plane is tangent to a sphere if and only if it is perpendicular to a radius at its extremity. [M11, 12, 13.]

5. A straight line tangent to a circle of a sphere lies in a planc tangent to the sphere at the point of contact. [*]

6. The distances of all points of a circle on a sphere from its poles are equal. [H1.]

7. A point on the surface of a sphere, which is at the distance of a quadrant from each of two other points, not the extremities of a diameter, is the pole of the great circle passing through these points. [H3.]

8. A sphere may be inscribed in or circumscribed about any given tetrahedrou. [H5.]

9. A spherical angle is measured by the arc of a great circle described from its vertex as a pole and included between its sides (produced if necessary).

[K5.]

\section{Spherical Triangles and Polygons.}

(Every theorem stated here may also be stated as a theorem on polyhedral angles.)

I. Each side of a spherical triangle is less than the sum of the other two sides. [011 (b). See also (a).]

2. The sum of the sides of a spherical polygon is less than $360^{\circ}$. [012 (b). See also (a). $]$

3. The sum of the angles of a spherical triangle is greater than $180^{\circ}$ and less than $540^{\circ}$. [K8.]

4. If $A^{\prime} B^{\prime} C^{\prime}$ is the polar triangle of $A B C$, then, reciprocally, $A B C$ is the polar of $A^{\prime} B^{\prime} C^{\prime}$. [K6.] 
5. In two polar triangles each angle of the one is the supplement of the opposite side in the other. [K7.]

6. Fertical spherical triangles are symmetrical and equivalent. $[\mathrm{C} 8, \mathrm{H} 6$.

7. Two triangles ${ }^{47}$ on the same sphere are either congruent or symmetrical if

$$
\begin{aligned}
& \mathrm{a}=\mathrm{a}^{\prime} \quad \mathrm{b}=\mathrm{b}^{\prime} \quad \mathrm{c}=\mathrm{c}^{\prime} \quad[\text { B2 (b). See also (a). }] \\
& \text { or } a=a^{\prime} \quad b=b^{\prime} \quad \mathbf{C}=\mathbf{C}^{\prime} \quad[B 3(b) \text {. See also (a). }] \\
& \text { or } a=a^{\prime} \quad B=B^{\prime} \quad C=C^{\prime} \quad[B 4(b) \text {. See also (a). }] \\
& \text { or } \mathbf{A}=\mathbf{A}^{\prime} \quad \mathbf{B}=\mathbf{B}^{\prime} \quad \mathbf{C}=\mathbf{C}^{\prime} \quad[B 5 \text { (b). See also (a). }]
\end{aligned}
$$

s. Either of the equations $a=b, \mathrm{~A}=\mathrm{B}$, is a consequence of the other. [*]

\section{Mensuration.}

(The relation between the areas and volumes of similar solids may be treated as corollaries in individual cases. See preface, article 3 . It is understood that certain statements concerning limits may be assumed either explicitiy or implicitly; these are not stated as theorems. See Q3, 4, 9, R9, 10.)

1. An oblique prism is equivalent to a right prism whose base is a right section of the oblique prism and whose altitude is a lateral edge of the oblique prism. [C4.]

2. A plane passed through two diagonally opposite edges of a parallelepiped divides it into two equivalent triangular prisms. [C6.]

3. The lateral area of a prism is the product of a lateral edge and the perimeter of a right section. [Q1.]

(Corollary of Plane Geometry.)

4. The lateral area of a regular pyramid is one-half the product of the slant height and the perimeter of the base. [Q2.]

(Corollary of Plane Geometry.)

5. The lateral area of a right circular cylinder is the product of the altitude and the circumference of the base; i. e., $s=2 \pi r h$. [Q5.]

6. The lateral area of a right circular cone is one-half the product of the slant height and the circumference of the base; i. e., $s=\pi r l . \quad[\mathbf{Q 6}$.

7. The lateral area of a frustum of a regular pyramid is one half the product of the slant height and the sum of the perimeters of the bases. [Q7.]

8. The lateral area of a frustum of a right cireular cone is one half the product of the slant height and the sum of the circumferences of the bases. [Q8 (a).]

4iThe same notation is used as in the plane triangles. 
9. The area of a zone is the product of its altitude and the circumference of a great circle; i. e., $s=2 \pi r h$. [Q10.]

(Lemma for 10 below.)

10. The area of a sphere is the product of its diameter and the circumference of a great circle; i. e., $s=4 \pi^{2}$. [Q11.]

11. The area of a lune is to the surface of a sphere as the angle of the lune is to $360^{\circ}$. [Q12.]

12. The area of a spherical triangle is to the area of the sphere as its spherical excess is to $720^{\circ}$. [Q13.]

13. The volume of a rectangular parallelepiped is the product of its three dimensions. $[R 1,2,3$.

(This may be taken as a definition.)

14. The volume of any parallelepiped is the product of its kase and altitude. [C5, R4.]

5. The volume of any prism is the product of its base and its altitude. [R5, 6.]

I6. The volume of any pyramid is one third the product of its base and its altitude. [C7, R7, 8.]

17. The volume of a circular cylinder is the product of its base and its altitude, i. e., $v=\pi r^{2} h$. [R11.]

I8. The volume of a circular cone is one third the product of its base and its altitude; i. e., $v=\mathrm{T} / 3 \pi r^{2} h$. [R12.]

I9. The volume of a spherical sector is one third the product of the radius and the zone which is its base; i. e., $v=$ $2 / 3 \pi \mathrm{r}^{2} \mathrm{~h}$. [R15.]

20. The volume of a sphere is one third the product of its radius and its area; i. $e ., v=4 / 3 \pi r^{2}$. [R16.]

(The wording suggests a proof, but that proof is by no means prescribed. The wording is convenient, the proof may even preferably follow 21 below:)

The following two theorems, while not thought by the committee to be indispensable, offer both for student and teacher an outlook for that larger view of geometry and of mathematics as a whole which is very desirable. They forecast important principles in future mathematical courses; they are capable of the nost practical direct applications; they offer a possibility of organizing and retaining the important mensuration formulæ given above.

21. If two solids contained between the same parallel planes are such that their sections by a plane parallel to those planes are equal in area, the two solids have the same volume. [*]

("Caralieri's Theorem." Formal proof should not be given.)

22. The volume of any sphere, cone, cylinder, pyramid, or prism, or of any frustum of one of these solids intercepted by two parallel planes, is given by the formula $v=1 / 8 h(t+4 m+b)$, where $t$ is the area of the upper base, $b$ that of the lower base, $m$ that of a base midway between the two, 
and where $h$ is the perpendicular distance between the two parallel planes. [See R13, 14.]

(This formula also applies to any so-called prismatoid; it is conveniently useful in practical affairs. It should not be proved for the general case, but each separate solid mentioned above, numbers 1 to 20 , can be shown to conform to this rule, by a direct check.)

\section{CONCLUSION.}

It should be said that the members of the committee are not entirely agreed as to certain minor details of this report. For example, some would place among the exercises certain propositions now in small type; others would prefer to put some theorems in black faced type which are now in italics; others would prefer three types of propositions instead of four; and some would modify certain postulates and would consider as postulates or as propositions to be demonstrated certain theorems included in the list of those requiring only informal proof. The committee does not regard these minor matters of any great consequence, and therefore wishes to be considered as approving the spirit and general tenor of the report, rather than as giving individual sanction to all such detaịls.

Herbert L. Sladght, Chairman,

The University of Chicago, Chicago, Ill.

WILLIAM BETZ,

East High School, Rochester, N. Y.

EDWARD I. Brown,

North High School, Denver,

Charles L. Bouton,

Harvard University, Cambridge, Mass.

Florian Cajori,

Colorado College, Colorado Springs, Colo.

WILLIAM FUILER,

Mechanic Arts High School, Boston, Mass.

WaLter W. Hart,

University of Wisconsin, Madison, Wis.

HERBERT E. HAw Kes,

Columbia University, New York City.

EARLE R. HEDRICK,

University of Missouri, Columbia, Mo.

FrEDERICK E. Newton,

Phillips Academy, Andover, Mass.

HENRY L. RIETz,

University of Illinois, Urbana, Ill.

ROBERT L. SHORT,

Technical High School, Clepeland, Ohio.

Dayid Eugene SMirh,

Teachers College, Columbia University, New York City.

Eugene R. SMith,

Polytechnic Preparatory School, Brooklyn, N. Y.

MABei Sykes,

Bowen High School, Chicago, Ill. 